\title{
Genome-Wide TDT Analysis in French-Canadian Families with Tourette Syndrome
}

\author{
Jean-Baptiste Rivière, Judith St-Onge, Claudia Gaspar, Sabrina Diab, Yves Dion, Paul \\ Lespérance, Geneviève Tellier, François Richer, Sylvain Chouinard, Marie-Pierre Dubé, \\ Guy A. Rouleau, and the Montreal Tourette Study Group
}

Can. J. Neurol. Sci. 2010; 37: 110-112

Tourette syndrome (TS) is a complex neurodevelopmental disorder with an estimated prevalence of $1 \%{ }^{1}$ that is characterized by motor and vocal tics as well as psychiatric comorbidities, such as obsessive-compulsive disorder (OCD) and attention deficit hyperactivity disorder (ADHD) ${ }^{2-4}$ Despite a strong genetic contribution, ${ }^{5,6}$ no common variants have been clearly associated with the disorder, possibly because of allelic and non-allelic genetic heterogeneity. Several candidate genes involved in dopaminergic neurotransmission have been analyzed based on the observation that neuroleptics are used to treat TS patients. Positive association results between TS and some of these genes have been reported. ${ }^{7-9}$ However, because other studies failed to replicate these results, the role played by dopaminergic in TS remains unclear. ${ }^{10-12}$ Founder populations are known to offer several advantages for the gene-mapping of complex traits, as the reduced genetic heterogeneity of population isolates is thought to simplify the genetic background of complex traits and higher frequencies of specific mutations inherited from a common ancestor may be observed. Moreover, even if the number of implicated genes is not decreased, the allelic heterogeneity is decreased and the presence of a common haplotype that segregates among patients is more probable than within a heterogeneous population. ${ }^{13}$ For instance, the G2019S substitution in the LRRK2 gene accounts for $20-40 \%$ of NorthAfrican Arab and Ashkenazi patients with Parkinson disease. ${ }^{14,15}$ In the French-Canadian (FC) population, founder mutations in the BRCA1 and BRCA2 genes were identified in $40 \%$ of patients from families with a high risk of breast and ovarian cancer. ${ }^{16}$ For several reasons, the FC population of Quebec displays all the characteristics of a population isolate. An estimated 2,600 pioneers who settled in Quebec before 1680 account for two thirds of the modern FC gene pool and a vast majority of FC people have, as ancestors, approximately 7,000 individuals who immigrated to Quebec before 1760. Given that these founders rarely mixed with other immigrants over three centuries and that there was a sustained demographic growth in the FC population, the $\sim 6$ million FC individuals currently living in Quebec inherited most of their genes from a relatively small pool of founders. ${ }^{17}$

Based on a cohort of 217 FC trios (father, mother and proband) presenting with TS, the goal of this study was to assess the presence of frequent and highly penetrant alleles predisposing to TS in the FC population. Affected individuals and their relatives were recruited through the Montreal General
Hospital and the Sainte-Justine Hospital (Montreal). Experienced clinicians performed diagnostic evaluations of TS, chronic tics and ADHD using the Diagnostic and Statistical Manual of Mental Disorders-IV criteria. Obsessive-compulsive disorder was evaluated using the Yale-Brown ObsessiveCompulsive Scale. ${ }^{18}$ We obtained approval from the ethics committee of our institution, as well as informed consent from all participants. The DNA was extracted from whole blood using standard procedures. For the purpose of the family-based genome-wide association analysis, we selected 95 trios among the 217 nuclear families recruited. To avoid the inclusion of sporadic cases, we selected only patients with a positive familial history of TS, tics or comorbid psychiatric disorders. Among the 95 selected TS cases, 73 were male, 31 presented with OCD and 53 received a diagnosis of ADHD. The DNA samples from the probands and their parents were sent to the deCODE genotyping service (http://www.decode.com/ genotyping). Five hundred fifty-one highly polymorphic microsatellites covering the 22 autosomes and the $\mathrm{X}$ chromosome with an average marker density of 8 centimorgans were genotyped using standard methods. The successful genotyping rate was $95.8 \%$ and Mendelian inconsistencies were systematically removed. We performed a family-based transmission disequilibrium test (TDT) using the Family-Based Association Test (FBAT) program, version 2.0.2C.19,20 The TDT determines whether an excess or a lack of transmission of alleles to the affected offspring occur. Multiallelic tests of association were performed for each marker. The additive genetic model was applied, as it often performs best, even when the genetic model is not additive. ${ }^{21}$ The significance level was set at 0.00009 after Bonferroni correction for multiple testing. Employing the TDT Power Calculator software, we assessed the statistical power of the TDT from 95 trios $^{22}$. Table 1 displays the number of trios

From the The Centre of Excellence in Neuromics, CHUM Research Center and the Department of Medicine (JBR, JSO, CG, SD, YD, PL, SC, GAR), University of Montreal; CHU Sainte-Justine, Department of Psychiatry (GT); Université du Québec à Montréal, Department of Psychology (FR); Research Centre of the Montreal Heart Institute, Department of Statistical Genetics (MPD), Montreal, Quebec, Canada. Received June 9, 2009. Final Revisions Submitted August 5, 2009. Correspondence to: Guy A. Rouleau, Ste-Justine Hospital Research Center and the Center of Excellence in Neuromics, University of Montreal, 1560, rue Sherbrooke Est, De-Seve Pavillion, room Y-3616-2, Montréal, Québec, H2L 4M1, Quebec, Canada. 
Table 1: Computation of the statistical power: Number of trios necessary to achieve $90 \%$ power in the TDT

\begin{tabular}{cccccc}
\hline & & \multicolumn{4}{c}{ Linkage disequilibrium coefficient } \\
& & 1.0 & 0.8 & 0.6 & 0.4 \\
\hline & 0.4 & 50 & 81 & 145 & 325 \\
Frequency of the disease & 0.2 & 66 & 105 & 187 & 420 \\
susceptibility allele & 0.1 & 87 & 138 & 247 & 552 \\
& 0.05 & 126 & 198 & 353 & 789 \\
\hline
\end{tabular}

The frequency of marker allele segregating with the disease susceptibility allele is 0.4 , and the recombination fraction between the marker locus and the disease susceptibility locus is 0 . Genotype penetrances for $A A$ (homozygotes for the susceptibility allele), $A a$ (heterozygotes for the susceptibility allele), and $a a$ (non-carriers of the susceptibility allele) are $0.98,0.28$ and 0.01 , respectively.

estimated to be necessary to achieve $90 \%$ statistical power with a significance level of 0.00009 , according to variable disease susceptibility allele frequencies and degrees of linkage disequilibrium between a marker and a disease susceptibility locus. Although none of the markers reached the significance level for association, we obtained $P$ values $<0.005$ for four markers (D7S2485, D13S271, D15S1016 and D19S605; Figure and Table 2). None of the markers are located in any of the chromosomal loci proposed to be linked to TS by the Tourette Syndrome Association International Consortium for Genetics. ${ }^{23,24}$ To attempt to replicate our findings, the four markers were genotyped in the remaining $122 \mathrm{FC}$ trios from our initial cohort of $217 \mathrm{FC}$ trios with TS. Unfortunately, the analysis of the genotype data from the $217 \mathrm{FC}$ trios revealed that none of the markers remained associated with TS (Table 2).

Our data suggest that common and highly penetrant founder mutations are unlikely to predispose to TS in the FC population. Our inability to replicate the association when the whole TS cohort was included in the analysis suggests that the peaks observed in the initial analysis were likely false-positive signals, which is strengthened by the fact that no single marker reached the genome-wide significance threshold. One may argue that the lack of replication of the association in the whole cohort may stem from the fact that we selected the 95 trios based on positive familial history of TS, and that the 122 remaining trios may comprise a significant proportion of non-genetic cases of TS. This is unlikely, as the vast majority of the cases in our cohort have first-degree relatives with TS, tics, ADHD or OCD, which indicates that sporadic cases with TS are the exception, rather than the rule. Our results suggest that, even in the case of a founder population (such as the FC), the genetically complex nature of TS renders this type of analysis powerless; the lack of significant association results in the analysis presented here may be explained by the complex inheritance pattern of TS and its comorbidities $^{25}$, which have a great negative impact on familybased studies. We are aware that our study presents several limitations, which include the relatively small number of families and the low marker density of the genome-wide scan. As presented in Table 1, statistical power computation indicates that our sample size was probably large enough to detect an allele segregating with a founder mutation carried by 20 to $40 \%$ of patients, but the study was most likely underpowered for founder mutation frequencies of $10 \%$ or less, unless the marker allele was in perfect linkage disequilibrium with a founder mutation. A high-density single nucleotide polymorphism genome-wide association study using a large cohort of patients may be necessary to verify whether TS originates from the combined effect of several common and low penetrant alleles. It would also

Table 2: TDT analysis (FBAT v.2.0.2C) of the four markers exhibiting a $P$ value $<0.005$, and association results in the $217 \mathrm{FC}$ TS trios

\begin{tabular}{ccccc}
\hline Marker & Chr & Position (bp) & $\begin{array}{c}95 \text { trios } \\
P \text { value }\end{array}$ & $\begin{array}{c}217 \text { trios } \\
P \text { value }\end{array}$ \\
\hline D7S2485 & $7 \mathrm{q} 21.11$ & $83,997,436$ & 0.000595 & 0.451994 \\
D13S271 & $13 \mathrm{q} 31.1$ & $84,278,764$ & 0.003924 & 0.325128 \\
D15S1016 & $15 \mathrm{q} 21.3$ & $51,320,121$ & 0.000341 & 0.234887 \\
D19S605 & $19 \mathrm{q} 13.42$ & $60,443,625$ & 0.003598 & 0.163923 \\
\hline
\end{tabular}

"Chr" refers to the chromosome band. The position of markers is in base pairs (bp) and was derived from the publicly available human genome assembly (UCSC Genome Browser). 


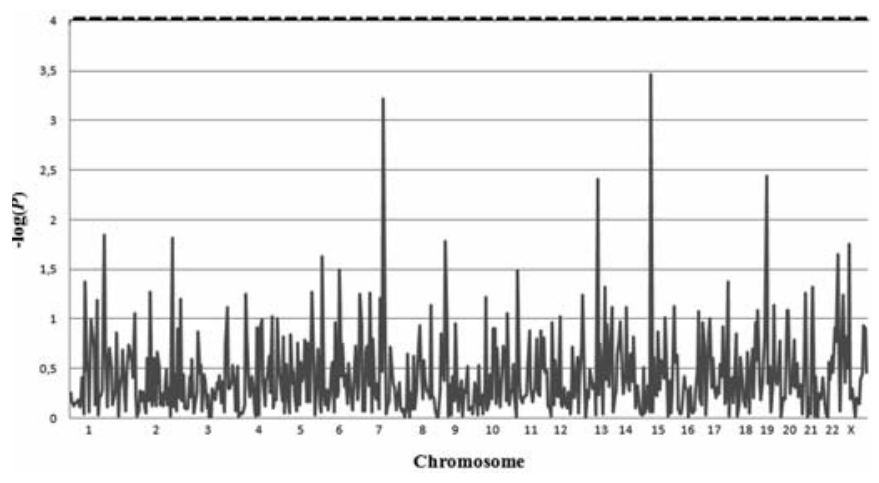

Figure: Genome-wide TDT analysis using FBAT (v.2.0.2C) in 95 FC TS trios. TDT analysis of the 551 microsatellite markers is represented by $-\log P$ values. The dotted line indicates the genome-wide significance level, which was set at 0.00009 after correction for multiple testing.

be feasible to use ADHD and OCD diagnoses as covariates, or to analyze subgroups of TS patients separately, based on their comorbidities. However, this approach would necessitate large cohorts of patients and would reduce the statistical power because of multi-test adjustments. If the genetic model for TS is that many cases are caused by individually rare, but highly penetrant mutations, this approach will not be successful, and a whole-genome resequencing analysis or large-scale screening of candidate genes will be warranted. Finally, this study illustrates the difficulty in identifying susceptibility genes for neuropsychiatric disorders and emphasizes the necessity to develop new gene-mapping approaches for these complex disorders.

\section{ACKNOWLEDGEMENTS}

This work was supported by the Tourette Syndrome Association. The authors thank the families for their collaboration. JBR is supported by the Canadian Institutes of Health Research.

Members of the Montreal Tourette Study Group: Maryse Charest, Van Chau, Véronique Desbeaumes, Marie-Hélène Dion, Michel Duplessis, Claire Girard, Daphnée Handanos, Nicolas Jodoin, Didier Jutras Assouad, Martin Lemay, Albert Ng, Myriam Srour, Marc Thibault, Isabelle Tremblay, Mijouk Vézina.

\section{REFERENCES}

1. Robertson MM. The prevalence and epidemiology of Gilles de la Tourette syndrome. Part 1: the epidemiological and prevalence studies. J Psychosom Res. 2008;65(5):461-72.

2. Definitions and classification of tic disorders. The Tourette Syndrome Classification Study Group. Arch Neurol. 1993;50 (10):1013-6.

3. Peterson BS, Pine DS, Cohen P, Brook JS. Prospective, longitudinal study of tic, obsessive-compulsive, and attention-deficit/ hyperactivity disorders in an epidemiological sample. J Am Acad Child Adolesc Psychiatry. 2001;40(6):685-95.

4. Robertson MM. Tourette syndrome, associated conditions and the complexities of treatment. Brain. 2000;123(Pt 3):425-62.

5. Pauls DL, Cohen DJ, Heimbuch R, Detlor J, Kidd KK. Familial pattern and transmission of Gilles de la Tourette syndrome and multiple tics. Arch Gen Psychiatry. 1981;38(10):1091-3.
6. Price RA, Kidd KK, Cohen DJ, Pauls DL, Leckman JF. A twin study of Tourette syndrome. Arch Gen Psychiatry. 1985;42(8):815-20.

7. Gade R, Muhleman D, Blake H, MacMurray J, Johnson P, Verde R, et al. Correlation of length of VNTR alleles at the X-linked MAOA gene and phenotypic effect in Tourette syndrome and drug abuse. Mol Psychiatry. 1998;3(1):50-60.

8. Grice DE, Leckman JF, Pauls DL, Kurlan R, Kidd KK, Pakstis AJ, et al. Linkage disequilibrium between an allele at the dopamine D4 receptor locus and Tourette syndrome, by the transmissiondisequilibrium test. Am J Hum Genet. 1996;59(3):644-52.

9. Diaz-Anzaldua A, Joober R, Riviere JB, Dion Y, Lesperance P, Richer F, et al. Tourette syndrome and dopaminergic genes: a family-based association study in the French Canadian founder population. Mol Psychiatry. 2004;9(3):272-7.

10. Brett PM, Curtis D, Robertson MM, Gurling HM. Exclusion of the 5-HT1A serotonin neuroreceptor and tryptophan oxygenase genes in a large British kindred multiply affected with Tourette's syndrome, chronic motor tics, and obsessive-compulsive behavior. Am J Psychiatry. 1995;152(3):437-40.

11. Hebebrand J, Nothen MM, Ziegler A, Klug B, Neidt H, Eggermann $\mathrm{K}$, et al. Nonreplication of linkage disequilibrium between the dopamine D4 receptor locus and Tourette syndrome. Am J Hum Genet. 1997;61(1):238-9.

12. Comings DE, Gonzalez N, Wu S, Gade R, Muhleman D, Saucier G, et al. Studies of the $48 \mathrm{bp}$ repeat polymorphism of the DRD4 gene in impulsive, compulsive, addictive behaviors: Tourette syndrome, ADHD, pathological gambling, and substance abuse. Am J Med Genet. 1999;88(4):358-68.

13. Varilo T, Peltonen L. Isolates and their potential use in complex gene mapping efforts. Curr Opin Genet Dev. 2004;14(3):316-23.

14. Lesage S, Durr A, Tazir M, Lohmann E, Leutenegger AL, Janin S, et al. LRRK2 G2019S as a cause of Parkinson's disease in North African Arabs. N Engl J Med. 2006;354(4):422-3.

15. Ozelius LJ, Senthil G, Saunders-Pullman R, Ohmann E, Deligtisch A, Tagliati M, et al. LRRK2 G2019S as a cause of Parkinson's disease in Ashkenazi Jews. N Engl J Med. 2006;354(4):424-5.

16. Tonin PN, Mes-Masson AM, Futreal PA, Morgan K, Mahon M, Foulkes WD, et al. Founder BRCA1 and BRCA2 mutations in French Canadian breast and ovarian cancer families. Am J Hum Genet. 1998;63(5):1341-51.

17. Heyer E, Tremblay M. Variability of the genetic contribution of Quebec population founders associated to some deleterious genes. Am J Hum Genet. 1995;56(4):970-8.

18. Goodman WK, Price LH, Rasmussen SA, Mazure C, Fleischmann RL, Hill CL, et al. The Yale-Brown Obsessive Compulsive Scale. I. Development, use, and reliability. Arch Gen Psychiatry. 1989;46(11):1006-11.

19. Laird NM, Horvath S, Xu X. Implementing a unified approach to family-based tests of association. Genet Epidemiol. 2000;19 Suppl 1:S36-42.

20. Rabinowitz D, Laird N. A unified approach to adjusting association tests for population admixture with arbitrary pedigree structure and arbitrary missing marker information. Hum Hered. 2000;50(4):211-23.

21. Horvath $\mathrm{S}, \mathrm{Xu} X$, Laird NM. The family based association test method: strategies for studying general genotype--phenotype associations. Eur J Hum Genet. 2001;9(4):301-6.

22. Chen WM, Deng HW. A general and accurate approach for computing the statistical power of the transmission disequilibrium test for complex disease genes. Genet Epidemiol. 2001;21(1):53-67.

23. A complete genome screen in sib pairs affected by Gilles de la Tourette syndrome. The Tourette Syndrome Association International Consortium for Genetics. Am J Hum Genet. 1999;65(5):1428-36.

24. Genome scan for Tourette disorder in affected-sibling-pair and multigenerational families. Am J Hum Genet. 2007;80(2):265-72.

25. McMahon WM, van de Wetering BJ, Filloux F, Betit K, Coon H, Leppert M. Bilineal transmission and phenotypic variation of Tourette's disorder in a large pedigree. J Am Acad Child Adolesc Psychiatry. 1996;35(5):672-80. 